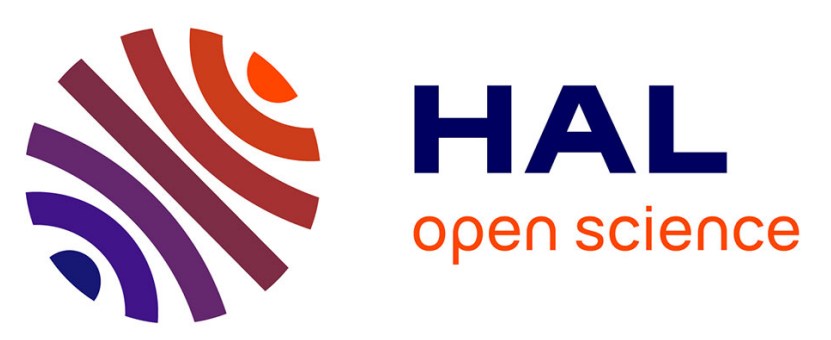

\title{
Cholesteric liquid crystals with a helical pitch gradient: Spatial distribution of the concentration of chiral groups by Raman mapping in relation with the optical response and the microstructure
}

\author{
M. Belalia, Michel Mitov, C. Bourgerette, A. Krallafa, M. Belhakem, D.
}

Bormann

\section{To cite this version:}

M. Belalia, Michel Mitov, C. Bourgerette, A. Krallafa, M. Belhakem, et al.. Cholesteric liquid crystals with a helical pitch gradient: Spatial distribution of the concentration of chiral groups by Raman mapping in relation with the optical response and the microstructure. Physical Review E : Statistical, Nonlinear, and Soft Matter Physics, 2006, 37 (5), 10.1103/PhysRevE.74.051704 . hal-02887360

\author{
HAL Id: hal-02887360 \\ https://hal.science/hal-02887360
}

Submitted on 2 Jul 2020

HAL is a multi-disciplinary open access archive for the deposit and dissemination of scientific research documents, whether they are published or not. The documents may come from teaching and research institutions in France or abroad, or from public or private research centers.
L'archive ouverte pluridisciplinaire HAL, est destinée au dépôt et à la diffusion de documents scientifiques de niveau recherche, publiés ou non, émanant des établissements d'enseignement et de recherche français ou étrangers, des laboratoires publics ou privés. 


\title{
Cholesteric liquid crystals with a helical pitch gradient: Spatial distribution of the concentration of chiral groups by Raman mapping in relation with the optical response and the microstructure
}

\author{
M. Belalia, ${ }^{1,3}$ M. Mitov, ${ }^{2}$ C. Bourgerette, ${ }^{2}$ A. Krallafa, ${ }^{3}$ M. Belhakem, ${ }^{4}$ and D. Bormann ${ }^{1}$ \\ ${ }^{1}$ Centre de Recherches sur les Matériaux à Haute Température, CNRS-CRMHT UPR4212, \\ Université d'Orléans, 1D, avenue de la Recherche Scientifique 45071 ORLEANS cedex 2, France \\ ${ }^{2}$ Centre d'Elaboration de Matériaux et d'Etudes Structurales, CNRS-CEMES UPR8011, BP 94347, \\ 31055 TOULOUSE cedex 4, France \\ ${ }^{3}$ Laboratoire de Chimie-Physique Macromoléculaire, Département de Chimie, Université d'Oran Es-Senia, 31100 ES-SENIA, Algeria \\ ${ }^{4}$ Laboratoire SEA2M, Département de Chimie, Université de Mostaganem, BP 227 MOSTAGANEM 27000, Algeria
}

(Received 25 July 2006; published 8 November 2006)

\begin{abstract}
Cholesteric liquid crystals (CLC) selectively reflect light when the helical pitch is of the order of the wavelength of the incident beam propagating along the helix axis. The wavelength bandwidth, related to the optical anisotropy, is typically limited to a few tens of nanometers in the visible part of the spectrum, which is insufficient for applications such as white-or-black polarizer-free reflective displays and smart windows for the control of the solar light and heat. A way to make cholesteric films reflecting in a broad wavelength band consists in associating various cholesteric pitches in the same film. In this work, it is shown how a study by confocal micro Raman spectrometry mapping makes it possible to have access to information accounting for the local organization of CLCs in the case of graded pitch materials. These investigations will be correlated to the optical response and the transverse microstructure of the CLC material as investigated by transmission electron microscopy. An accurate analysis of the vibrational behavior evolution of the $\mathrm{C}=\mathrm{O}$ can be correlated to the evolution of the populations of the chiral and achiral groups in the case of the interdiffusion of two CLC substances with various stoechiometries. Besides an easy measurement of the Raman spectrum gives the opportunity to quantify the relative ratio of the mesogenic species and thus to go up by a simple way to the pitch of the helical structure.
\end{abstract}

DOI: 10.1103/PhysRevE.74.051704

PACS number(s): 61.30.Eb, 42.70.Df, 36.20.Ng, 68.37.Lp

\section{INTRODUCTION}

\section{A. The selective light reflection in cholesteric liquid crystals}

Due to their helical structure, cholesteric liquid crystals (CLCs) selectively reflect the light $[1,2]$. When the incident light propagates essentially along the helical axis (i.e., when the CLC slab exhibits a planar Grandjean texture), a selective reflection occurs if the wavelength $\lambda_{0}$ is of the order of the pitch $p$ of the helix such that (at normal incidence):

$$
\lambda_{0}=n p,
$$

where $n$ is the average refractive index, $\left(n_{\|}+n_{\perp}\right) / 2\left(n_{\|}\right.$and $n_{\perp}$ are locally parallel and perpendicular to the molecular director). The reflection occurs within a bandwidth $\Delta \lambda$ $=\Delta n p$, where $\Delta n=n_{\|}-n_{\perp}$ is the birefringence. Inside this band an incident beam which propagates parallel to the helical axis is split into two circularly polarized components: one of which is simply transmitted whereas the other is totally reflected; the rotation of the reflected circularly polarized light agrees with the screw sense of the structure. Outside this band, a CLC affects neither the amplitude nor the state of polarization of the transmitted light.

\section{B. The broadening of the reflection bandwidth in cholesteric polysiloxane materials}

However huge the choice for pitch amplitude is, $\Delta n$ is typically limited to values below 0.4 for colorless organic materials. Consequently, $\Delta \lambda$ is commonly less than $100 \mathrm{~nm}$ in the visible spectrum and is more often equal to $50 \mathrm{~nm}$. The selectivity is desired for several families of applications-optical filters, thermal imaging, laser or paint technologies-, but a drawback lies in the fact that the known CLC filters have a reflection bandwidth that is too limited for specific purposes, like full-color or white-or-black reflective displays, broadband circular polarizers, or smart windows in buildings for which a control over the solar spectrum is desirable. The choice of polymer-stabilized CLCs elaborated under specific ways has offered the opportunity to increase the wavelength reflection bandwidth [3-7]. An earlier solution has consisted in the elaboration of a cholesteric layer with a pitch gradient; for producing such a structure, a UV-absorbing dye was used to create an intensity gradient of the UV-light through the thickness of a solid cholesteric polymer network [8] or a switchable CLC gel [9].

In an earlier work devoted to cholesteric liquid crystalline films in the glassy state, we described the elaboration procedure to obtain a material exhibiting a broadband pass for the light reflection in the visible spectrum [10]. Cholesteric polysiloxane oligomers were used [11]. These compounds are studied since a few years already and the various properties observed, in particular the selective reflection, are closely related to the pitch of the helical structure; it was established that the pitch, and consequently the reflection properties, may be adjusted according to the relative proportion between the chiral side chains (bearing the cholesterol group) and the achiral ones (bearing the nematogen group). By contrast to previous studies, the process we described 
depends on a thermal diffusion between two CLC oligomer films with different chiralities and the pitch gradient is the consequence of a concentration gradient which is then frozen by quenching [12]. Neither polymerization reaction nor photoinduced phase separation is involved. Besides, the reflector is a single-layer CLC film in the glassy state. $\Delta \lambda$ may simply be tuned by varying the annealing time and different optical properties (such as tunable bandwidths) are available. The pitch gradient was demonstrated and evaluated by transmission electron microscopy (TEM) investigations of cross sections $[13,14]$. Indeed, because the TEM technique produces reproducible and well contrasted images it is considered as one of the most suitable methods to explore the periodic structure of the studied material with a satisfactory accuracy. The origin of the contrast of the cholesteric substance, made of alternated bright and dark lines, is attributed to the change of orientation of the cholesteric molecules [15]. The optical behavior of these films has recently been related to theoretical studies [16]: it corresponds to the averaged contribution of a number of stochastic pitch variation profiles, due to transversal and longitudinal nonuniformities that develop in the helical structure.

When two CLC layers are associated in an appropriate manner, the environment of each molecule is changed and an apparent new pitch is obtained. Therefore, it is interesting to follow such a phenomenon from considerations taken into account the molecular vibrations.

\section{The scope of the present study}

Recent Raman analysis on CLCs mainly focused on the $\mathrm{C}=\mathrm{O}$ stretching mode behavior as a preferential witness of structural organization for various cholesteryl alkanoates [17], the molecular modeling of the constitutive molecules [18], or the diffraction characteristics of cholesteric gratings $[19,20]$. In this context, confocal Raman microspectrometry shows a huge potential to investigate the variation of the material composition within this original context of a cholesteric material with a structure gradient in the frozen state. In addition, Raman spectrometry experiments are highly sensitive to slight variations in chemical composition and/or crystallographic structure. It is also a noncontact and nondestructive technique which does not need any sample preparation. In many cases, therefore this allows the preservation of small traces of evidence for further analysis of the same specimen. Besides, only minimal quantities of material are required to perform a complete analysis resulting from the high spatial resolution of the confocal system. In this work, we will show how a study by confocal micro Raman spectrometry mapping makes it possible to have access to information accounting for the local organization of CLCs. These investigations will be correlated to the optical response and the transverse microstructure of the CLC material as investigated by transmission electron microscopy. In an original way, the three sets of investigations are made on the one and only sample.

\section{EXPERIMENTAL}

\section{A. Materials}

Cyclic siloxanes substituted with mesogenic groups, which are connected to the backbone by aliphatic spacers,

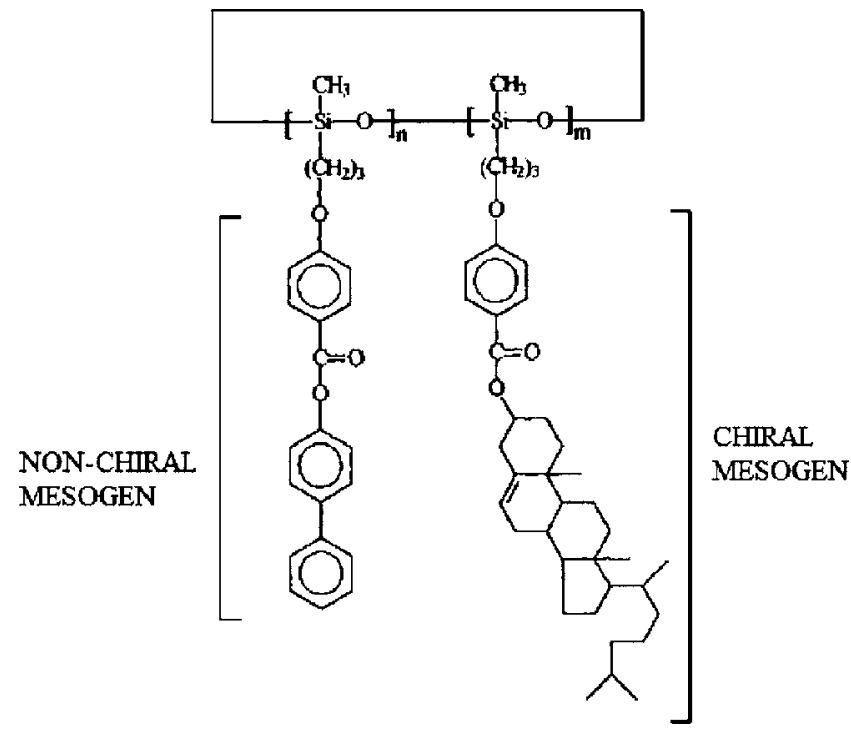

FIG. 1. General formula of a cholesteric polysiloxane oligomer. $n$ and $m$ are respectively the number of achiral and chiral side chains in the oligomer molecule $(n+m=5$ to 7$)$.

exhibit liquid crystalline phases as the classic calamitic liquid crystals [11]. Such side chain cyclic siloxane oligomers were used in the present study. The side chains were of two types - chiral (bearing the cholesterol group) and achiral (with a nematogenic group) - and connected to the cyclic siloxane via a $\left(\mathrm{CH}_{2}\right)_{3} \mathrm{O}$ spacer (Fig. 1). Typically a siloxane cycle contains $n+m=5$ to 7 monomers where $n$ and $m$ are the number of achiral and chiral moieties, respectively.

On a glass or plastic plate, the compound shows typical iridescent colors ranging from blue to red simply tuned by the molar percentage of chiral mesogens in the oligomer molecule, which varies from 50 to $31 \%$. The cholesteric phase appears by annealing in the range defined by the glass transition temperature $\left(40-50^{\circ} \mathrm{C}\right)$ and the isotropic transition temperature $\left(180-210^{\circ} \mathrm{C}\right)$. These materials are mutually miscible and can be very easily quenched at room temperature so that the cholesteric order is permanently frozen within a solid film.

In a first step we will study by Raman spectrometry two different films, one reflecting in the blue range - named SB, with $50 \%$ chiral side chains $(n=m)$ and one reflecting in the red range - named SR - with $31 \%$ of chiral side chains $[0.31=m /(n+m)]$. The pitch value of these materials was previously reported [16]: it is about $475-480 \mathrm{~nm}$ for SR and 290-295 $\mathrm{nm}$ for SB.

\section{B. Broadband reflector design}

The material design is based on an anisotropic diffusion between CLCs with different chiralities. The basic idea consists in making jointly forward in the cholesteric phase a blue film (SB) and a red film (SR) what will involve their interdiffusion and the formation of a film (named SR-SB) with a variable pitch in a direction perpendicular to the film surfaces. The experimental procedure is summarized below into four steps. 


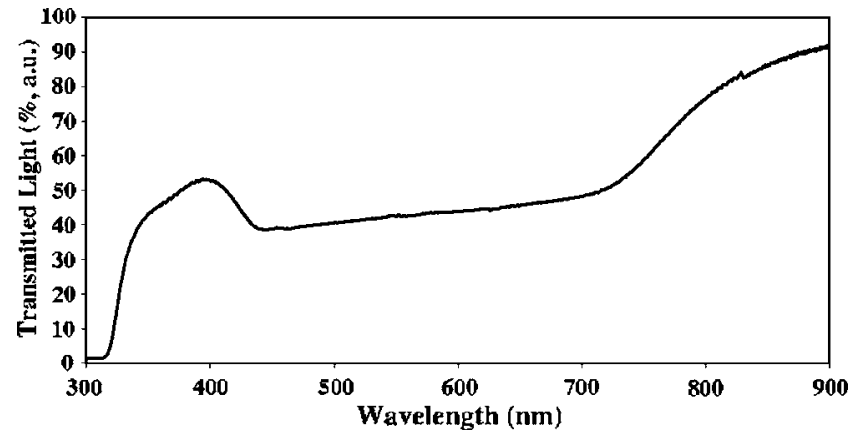

FIG. 2. Variation of the transmitted light as a function of the wavelength of the broadband cholesteric reflector used in the present study (the annealing time of the pristine bilayer film was equal to $25 \mathrm{~min}$ ).

Step 1: $40 \mu \mathrm{m}$ thick films of blue and red compounds are spread with a handcoater onto two distinct $1 \mathrm{~mm}$ thick glass plates without any alignment layer. Semifree films are thus fabricated.

Step 2: $19 \mu \mathrm{m}$ calibrated spacers are disposed between the plates but not in contact with the semifree films. In such a way a sandwich-cell is made and the layers are in contact. The cell is sealed with glue.

Step 3: The cell is kept at $85^{\circ} \mathrm{C}$. This temperature corresponds to a stable cholesteric phase for which the materials are viscous. Consequently, a diffusion process between the red and blue compounds - and therefore a concentration gradient-may occur in a direction perpendicular to the plane of films. Since these materials belong to the same molecule family, high miscibility is reached.

Step 4: After such an annealing process, the cell is swiftly put on a metallic substrate at room temperature. The macroscopic characteristics of the film evolve from a viscous to a glassy state. Such a cooling prevents crystallization and preserves the cholesteric structure. The quenching procedure is not affected by the fact that the cholesteric compounds have slightly different glassy transitions (due to the different number of cholesterol-containing side chains), since room temperature $\left(20-25^{\circ} \mathrm{C}\right)$ is well below their transition temperatures and the quenching time is sufficiently quick.

\section{Spectrophotometry}

The spectral characteristics of materials are obtained at room temperature by unpolarized UV-visible light spectroscopy (UV-3100 Shimadzu spectrophotometer). Figure 2 shows the variation of the transmitted light with the wavelength in the case of the sample of the present study for which the annealing time is equal to $25 \mathrm{~min}$ (the spectra of individual films appear in Refs. [12] to [14] which the topic is to investigate the progressive variation of the reflection properties from the individual films to the monolayer film corresponding to a long annealing time). Nearly 50\% transmission comes from polarization selectivity. The sudden decrease of the transmittance below $400 \mathrm{~nm}$ is due to the cutoff wavelength for the glass. A broadband reflector is obtained: the light is reflected all over the visible spectrum and from about 435 to $720 \mathrm{~nm}$, which are the extreme values for the slightly tilted plateau. This is an expected consequence of the construction of a pitch gradient in the cholesteric superstructure. The tilt of the plateau may have different origins: the film may exhibit some wavelength-dependent light scattering (due to scattering losses between the domain walls), the number of helix turns per length unit is not the same from the blue part of the film to the red one (it may modify as a consequence the reflected light intensity), and the cut-off wavelength for the glass-close to the smaller wavelengths - affects the shape of the cell spectrum. To the naked eye, the film is colourless with a metallic aspect since it reflects $50 \%$ of the light over the visible spectrum. The forthcoming investigations (transmission electron microscopy and Raman spectrometry) will be carried out on this one and only sample.

\section{Transmission electron microscopy (TEM) investigations}

For the TEM experiments, the SR-SB film is embedded in an epoxy resin cured at $40{ }^{\circ} \mathrm{C}$. $90 \mathrm{~nm}$ thin slices were obtained with an ultramicrotome (Reichert Ultracut) in a direction crossing the sandwich film perpendicularly to the surface (cross sections) and retrieved on carbon-coated grids. The observations are carried out at room temperature by using a Philips CM12 electron microscope and in normal conditions; this means that the share of electrons received by the specimen is well higher than the critical share deleting the diffraction contrast, and that the images are produced by a thickness diffusion contrast subsequent to irradiation [15]. The contrast was enhanced by a slight defocus.

\section{E. Raman spectrometry}

The spectra were obtained with a DILOR (XY) 800 Raman spectrophotometer. We used an excitation wavelength of $514.5 \mathrm{~nm}$ and the beam was focused on the samples by using a microscope. The signal was collected through a premonochromator and a CCD detector was used. With this experimental configuration, a spectral resolution of the order of $2 \mathrm{~cm}^{-1}$ is obtained and the density of power was in the order of a few $\mathrm{mW}$ to avoid the destruction of the samples.

\section{RESULTS AND DISCUSSION}

Due to the fact that the SR-SB film is obtained from a SR and a SB film, we present on Fig. 3 the Raman spectra obtained for both individual films.

At first sight, the two spectra are of similar pace and do not seem to present significant differences. This result was expected since the two samples differ only by the relative proportion of the chiral and achiral groups characterized by $m$ and $n$.

On the vibrational behavior of the chiral and achiral groups we can notice that each one has specificities being able to be highlighted by vibrational spectrometry.

Differences between the SB and the SR spectra are perceivable in many spectral ranges and especially in the 1500 $1800 \mathrm{~cm}^{-1}$ one (insert Fig. 3) corresponding to stretching modes. The signal at $1600 \mathrm{~cm}^{-1}$ corresponds to the phenyl ring quadrant stretching modes and the little one at 


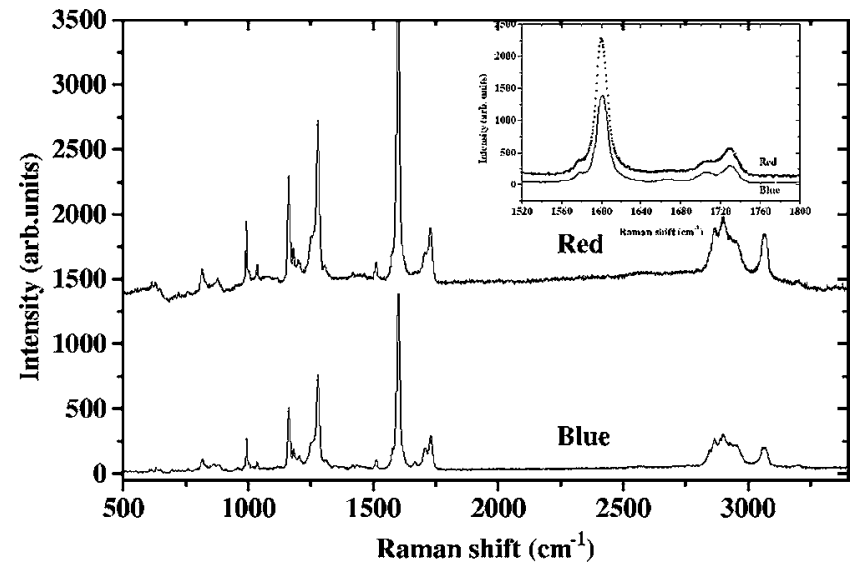

FIG. 3. Raman spectra of a pure "silicone blue" sample (SB) and a pure "silicone red" sample (SB) $(\lambda=514.5 \mathrm{~nm})$. Note the insert evidencing the existence of two stretching modes for the $\mathrm{C}=\mathrm{O}$ double bond at $1730 \mathrm{~cm}^{-1}$ and $1708 \mathrm{~cm}^{-1}$ respectively assigned to the achiral and the chiral groups.

$1667 \mathrm{~cm}^{-1}$ is ascribable to the double bond $\mathrm{C}=\mathrm{C}$ stretching mode. Carbonyl groups that differ in their chemical environment give modes appearing at $1730 \mathrm{~cm}^{-1}$ for carbonyl in the achiral group and at a lower frequency, $1708 \mathrm{~cm}^{-1}$ for carbonyl in the chiral group. The reason for this is that the $\mathrm{C}=\mathrm{O}$ double bond in the achiral group competes with the biphenyl in withdrawing electrons. On the other hand, in the chiral group the cholesteric moiety is an electron-donating group.

It is interesting to focus our attention on the relative intensity variation of the $1600 \mathrm{~cm}^{-1}$ band, due to the number of achiral chains in the SR sample. In fact, the number of achiral chains increases from the SB compound to the SR one and also the phenyls. This can be verified by the $1667 \mathrm{~cm}^{-1}$ peak intensity behavior which is different for SB and SR compounds. From the point of view of the present study, of peculiar interest is the behavior related to the stretching vibrations $\mathrm{C}=\mathrm{O}$ double bonds. Assuming that the frequency varies in relation with the number of achiral or chiral side chains, we deduce that the relative ratio between the $1708 \mathrm{~cm}^{-1}$ and the $1730 \mathrm{~cm}^{-1}\left(\frac{I_{\text {chiral }}(1708)}{I_{\text {achiral }}(1730)}\right)$ mode intensity is in fact a measurement for $n$ and $m$. In the case of our pure SB and SR samples we obtained respectively 0.80 and 0.60 (Lorentzian fitting).

By associating two cholesteric siloxane films (in agreement with the procedure described in Sec. II B we manufactured the SR-SB film (with an annealing time equal to $25 \mathrm{~min}$ ), and we obtained the TEM micrograph presented in Fig. 4.

The two components of the sandwich are clearly visible, the blue film and the red film, due to the different values of the periodicity (the distance between two dark or bright lines). This periodicity $(p / 2)$ is constant at the extremities of the cross section and the value is the one of the initial SB (left part) and SR (right part) films. The original ordered structure was preserved with a soft transition between the two parts. Figure 7 reports the diagram of the evolution of the pitch versus its distance from the film surface. It exhibits

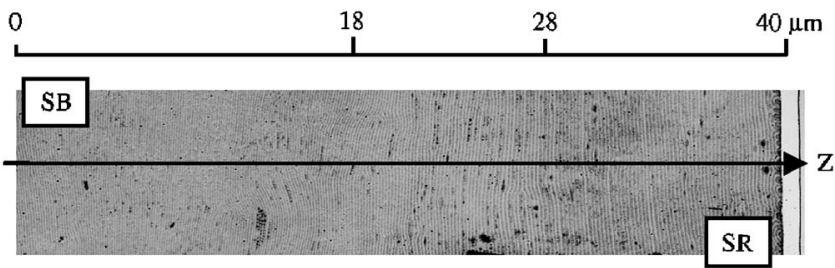

FIG. 4. TEM micrograph of the cross-section of a SB-SR film after annealing $(25 \mathrm{~min})$ and inter diffusion of SB and SR.

three regions corresponding, from the left to the right, to a linear behavior, a transition region (about $10 \mu \mathrm{m}$ thick) and a linear behavior again.

To see the distribution of the stoechiometries through the sample, we gradually acquire Raman spectra according to a trajectory going from the SB side towards the SR side through the zone of diffusion (interface) on the one and only material cut.

Like in the case of SB and SR phases (the extreme situations in our sample), which were characterized previously, this step will be used to follow at the same time the evolution of the various modes in terms of frequency and intensity.

We took a trajectory following the axis $Z$ in Fig. 4 and in the same experimental conditions than previously described.

Figure 5 exhibits a variation of the modes intensity for the carbonyls stretching through the sample. To analyze these spectra, we used adjustment of the peaks, centered roughly on $1740 \mathrm{~cm}^{-1}$ and $1710 \mathrm{~cm}^{-1}$. For that, we tried out three adjustment functions: Lorentzian, Gaussian, and Lorentzian/ Gaussian functions. Finally, we chose to work with the Lorentzian functions because this adjustment gives us the best estimate of mean square deviation. From there and without any constraint over neither the position nor the width with midheight of the modes, we obtained a data file corresponding to the position, the width at midheight, the intensity and the surface of the peaks. The midheight widths as well as the wave number were no relevant parameters and are invariant in the case of our material, so we planned to study the variation of the intensity and/or the surface of the peaks. Finally, the signal-to-noise ratio of the obtained spectra led us to estimate that the intensity is the most relevant parameter to compare the various phases.

From Fig. 6, the evolution of the relative intensities of the $\mathrm{C}=\mathrm{O}$ bands can be observed versus various points of the trajectory. First, we remark that the profile is divided into

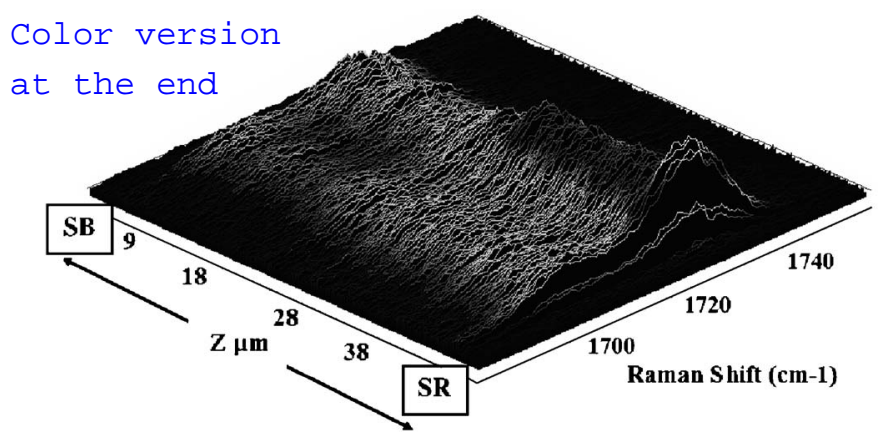

FIG. 5. Raman spectrometry map along the $Z$ axis defined in the carbonyl stretching range. 


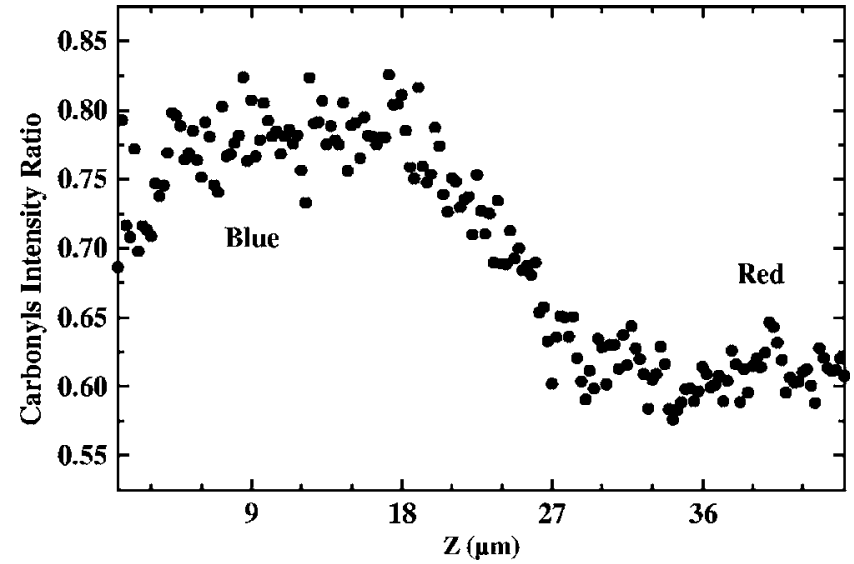

FIG. 6. Intensity ratio $\left(\frac{I_{\text {chiral }}(1708)}{I_{\text {achiral }}(1730)}\right)$ between the two carbonyl modes at $1708 \mathrm{~cm}^{-1}$ (chiral side chain) and $1730 \mathrm{~cm}^{-1}$ (achiral side chain). The SB and the SR sides of the SR-SB film give a ratio nearby constant with respective values of 0.8 and 0.6 . In the middle of the sample we observe a linear behavior corresponding to the inter-diffusion interface.

three parts. The left and the right parts present a constant intensity ratio of about 0.8 and 0.6 a.u. which respectively correspond to the SB and SR films. On the other hand, the ratio varies slightly and continuously in the intermediate region from about 0.78 a.u. to about 0.57 a.u. by presenting a linear distribution of the intermediate ratios.

Thus, it proves that the chiral side chains fraction gradually changes from the silicone blue to the silicone red film and this involves a gradual change of the cholesteric pitch, which depends on the amount of the cholesteric moiety in the siloxane molecule. This agrees with the TEM investigations for which the profile of the pitch along the $Z$ axis (defined in Fig. 4) shows the same trend (Figs, 6 and 7); however, a correlation between the pitch and the intensity ratios can be established and so, as we detail below, a quasi-direct measurement of the helical pitch by Raman spectroscopy can be made.

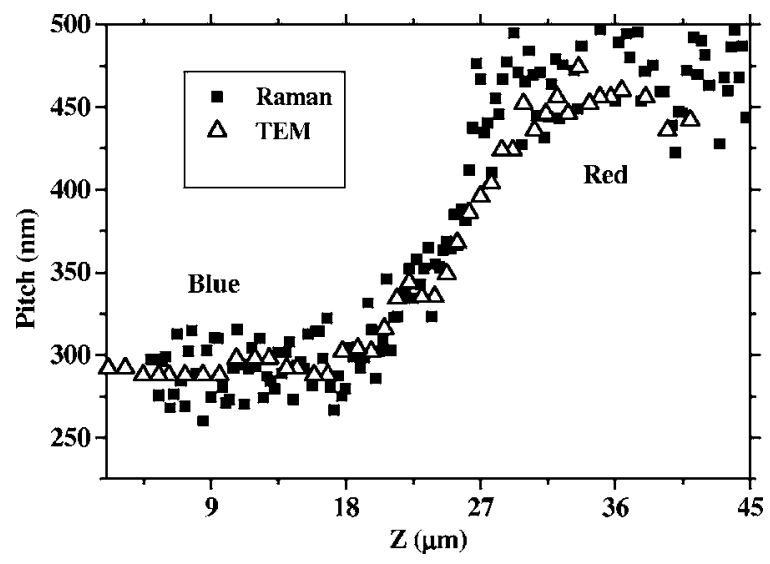

FIG. 7. Comparison of the pitch values obtained by TEM and Raman spectrometry. Both curves demonstrate the ability of Raman spectrometry to be a user-friendly tool to measure the cholesteric pitch. The interface is of the order of $10 \mu \mathrm{m}$.
It is shown that the reflection wavelength $\lambda_{[m /(m+n)]}$ is inversely proportional to the molar percentage of the chiral compound $C_{\text {chiral }}$ and is given by $[11,21]$

$$
\lambda_{[m /(m+n)]}=\frac{\text { const }}{C_{\text {chiral }}} .
$$

In the diffusion region, in which the concentration occurs, the concentration in chiral mesogens is given as a function of the respective concentration in the $\mathrm{SB}\left(C_{B}\right)$ and $\mathrm{SR}\left(C_{R}\right)$ as follows:

$$
C_{\text {chiral }}=x C_{B}+(1-x) C_{R} \Rightarrow x=\frac{C_{\text {chiral }}-C_{R}}{C_{B}-C_{R}},
$$

where $x$ is the chiral mesogens fraction in the diffusion region.

From Eqs. (1) and (2) we obtain

$$
\lambda_{[m /(m+n)]}=\frac{1}{a+x b}=\frac{1}{C_{r} / \text { const }+x\left(C_{B}-C_{r}\right) / \text { const }} .
$$

The $a$ and $b$ coefficients are determined from experimental data for SB $(x=1)$ and SR $(x=0)$ which are respectively $\lambda_{[m /(m+n)=0.5]}=450 \mathrm{~nm}$ and $\lambda_{[m /(m+n)=0.31]}=745 \mathrm{~nm}$. The extracted values are: $a=1.3810^{-3} \mathrm{~nm}^{-1}$ and $b=0.910^{-3} \mathrm{~nm}^{-1}$. Under the hypothesis that the mean refraction index is 1.55 for our samples we can deduce the pitch $p$ by

$$
p=\frac{1}{n(a+x b)}(\mathrm{nm}) .
$$

Considering that all the involved $\mathrm{C}=\mathrm{O}$ double bonds behave similarly, the intensity of the Raman diffusion is, in a first approximation, linearly dependent on the concentration in diffusing species, so we will be able to deduce $\mathrm{x}$ from the Raman intensity. From the experimental data we define $D$ by

$$
\begin{aligned}
D & =\frac{m}{m+n} \approx \frac{I_{\text {chiral }}(\mathrm{C}=\mathrm{O})}{I_{\text {chiral }}(\mathrm{C}=\mathrm{O})+I_{\text {achiral }}(\mathrm{C}=\mathrm{O})} \\
& =\frac{I_{\text {chiral }}(1708)}{I_{\text {chiral }}(1708)+I_{\text {achiral }}(1730)}
\end{aligned}
$$

and thus, by analogy with de concentration equation we deduce

$$
D_{\text {chiral }}=x D_{B}+(1-x) D_{R} \Rightarrow x=\frac{D_{\text {chiral }}-D_{R}}{D_{B}-D_{R}} .
$$

In Fig. 7 we present the results obtained for the pitch values by comparison with the ones issued from TEM investigations. We notice that we have a remarkable agreement between the results of both techniques and that these results validate our experimental procedure.

In the continuity of our work, we plan to continue our research on these systems by using the polarizability properties of the CLCs and the polarization of the vibrational modes. The use of polarized Raman spectrometry will give us access to the local order of the molecules what will give us important information as for the relative positioning of the chiral and achiral side chains. The access to these data will enable us to understand the intermolecular interactions and 
thus the mechanisms of diffusion in our materials.

On the potential to investigate the material properties, because the Raman mapping offers the opportunity to probe the concentration in chiral mesogens very locally, it opens the way to novel investigations to go into details about the diffusion mechanism between the individual films. As an example, the choice of the thickness of the blue and red films is of paramount importance for the existence and the propagation of the pitch gradient. Besides, the blue and red films have different viscosities. So it means that we could think to elaborate sandwich films from individual films with thinner thicknesses-or to choose different thicknesses from one film to the other one (case of asymmetric films) — to fabricate photonic bandgaps with different and tuneable filtering profiles. Then the origin of these optical properties could be elucidated from the investigation of the local concentration of chiral mesogens, and the time-resolved monitoring of the concentration variation during the annealing process may also be foreseen.

\section{CONCLUSION}

Broadening the light reflection bandwidth can be obtained by a simple thermal treatment of a cholesteric glassy film, with a reduced number of independent driving parameters. The helical pitches of these films were determinate by Raman microscopy and were found in excellent agreement with those estimated by the TEM. The procedure is easy to implement since the only condition is to be able to have two samples for calibrating and determining the couple $(a, b)$. Then the intensity measurements of the modes of vibration characteristic of the involved chemical groups (here chiral and achiral) leads to the determination of $x$ and the relative proportion of achiral and chiral side chains by the determination of the ratio $\frac{m}{m+n}$. As a consequence, we can calculate the pitch of the cholesteric phase such as: $p=\frac{1}{n(a+x b)}$. It has to be remark that this kind of measurement can easily be generalized to any kind of system for which the periodicity varies (structure-gradient materials) and is correlated to variations of relative chemical species concentration which have specific vibrational behaviors.

\section{ACKNOWLEDGMENTS}

The authors are grateful to Dr. F.-H. Kreuzer and Dr. E. Hanelt (Wacker Chemie $\mathrm{GmbH}$ ) for their kind support and the cholesteric compounds. E. Nouvet carried out the spectrophotometry measurements in Fig. 2.
[1] P. G. de Gennes and J. Prost, The Physics of Liquid Crystals (Oxford Clarendon Press, Oxford, 1993), p. 263.

[2] For a review: N. Boiko and V. Shibaev, Int. J. Polym. Mater. 45, 533 (2000).

[3] N. Leroux, W. J. Fritz, J. W. Doane, and L.-C. Chien, Mol. Cryst. Liq. Cryst. Sci. Technol., Sect. A 261, 465 (1995).

[4] J.-F. Li, B. Fan, and L. Li, SID Int. Symp. Digest Tech. Papers 30, 1066 (1999).

[5] C. Binet, M. Mitov, and M. Mauzac, J. Appl. Phys. 90, 1730 (2001).

[6] H. Guillard and P. Sixou, Liq. Cryst. 28, 933 (2001).

[7] M. Mitov, E. Nouvet, and N. Dessaud, Eur. Phys. J. E 15, 413 (2004).

[8] D. J. Broer, J. Lub, and G. N. Mol, Nature (London) 378, 467 (1995).

[9] R. A. M. Hikmet and H. Kemperman, Nature (London) 392, 476 (1998).

[10] M. Mitov, A. Boudet, and P. Sopéna, Eur. Phys. J. B 8, 327 (1999).

[11] F.-H. Kreuzer, N. Häberle, H. Leigeber, R. Maurer, J. Stohrer, and J. Weis, Organosilicon Chemistry III (WILEY-VCH, New
York, 1997), p. 566.

[12] C. Binet, M. Mitov, and A. Boudet, Mol. Cryst. Liq. Cryst. Sci. Technol., Sect. A 339, 111 (2000).

[13] A. Boudet, C. Binet, M. Mitov, C. Bourgerette, and E. Boucher, Eur. Phys. J. E 2, 247 (2000).

[14] M. Mitov, C. Binet, A. Boudet, and C. Bourgerette, Mol. Cryst. Liq. Cryst. Sci. Technol., Sect. A 358, 209 (2001).

[15] J. Pierron, A. Boudet, P. Sopéna, M. Mitov, and P. Sixou, Liq. Cryst. 19, 257 (1995).

[16] D. C. Zografopoulos, E. E. Kriezis, M. Mitov, and C. Binet, Phys. Rev. E 73, 061701 (2006).

[17] S. Bresson, D. Bormann, B. Khelifa, F. H. Reguig, and A. Krallafa, Vib. Spectrosc. 21, 27 (1999).

[18] J. Fayos, S. Sanchez-Cortes, C. Marco, and M. Perez-Mendez, J. Macromol. Sci., Phys. B4, 553 (2001).

[19] D. Subacius, S. V. Shiyanovskii, P. Bos, and O. D. Lavrentovich, Appl. Phys. Lett. 71, 3323 (1997).

[20] A. Y. G. Fuh, C. H. Lin, M. F. Hsieh, and C. Y. Huang, Jpn. J. Appl. Phys., Part 1 40, 1334 (2001).

[21] H. Ogawa, E. Stibal-Fischer, and H. Finkelmann, Macromol. Chem. Phys. 205, 593 (2004). 
